\title{
Detectability of Absorption and Reduced Scattering Coefficients in Frequency-Domain Measurements Using a Realistic Head Phantom
}

\section{Xiaofeng Zhang ${ }^{1, *}$ and Andrew Webb ${ }^{2}$}

1 Department of Radiology, Duke University Medical Center, DUMC 3808, Durham, NC 27710, USA

2 Department of Radiology, Leiden University Medical Center, Albinusdreef 2, 2333 ZA Leiden, The Netherlands; E-Mail: a.webb@lumc.nl

* Author to whom correspondence should be addressed; E-Mail: steve.zhang@duke.edu; Tel.: +1-919-257-8564; Fax: +1-919-684-2711.

Received: 7 November 2012; in revised form: 17 December 2012 / Accepted: 18 December 2012 / Published: 24 December 2012

\begin{abstract}
Detection limits of the changes in absorption and reduced scattering coefficients were investigated using a frequency-domain near-infrared system in a realistic head phantom. The results were quantified in terms of the maximum detectable depth for different activation volumes in the range of 0.8-20 microliters. The non-linear relation between the maximum detectable depth and the magnitude of changes in the absorption coefficient conform well with the Born approximation to the diffusion equation. The minimal detectable changes in the reduced scattering coefficient measured in terms of the phase signal were found to be approximately twice as large as that of the absorption coefficient using the AC signal for the same volume and at the same depth. The phase delay, which can be used to quantify the fast neuronal optical response in the human brain, showed a linear dependence on the reciprocal of the reduced scattering coefficient, as predicted by the Rytov approximation.
\end{abstract}

Keywords: near infrared; absorption; scattering; fast signal; detectability; sensitivity; frequency domain; phantom; human head 


\section{Introduction}

Near infrared (NIR) spectroscopy and imaging methods are increasingly important measurement tools in biology, neuroscience, and medicine because of their noninvasive nature, high chemical specificity, high temporal resolution, high versatility, and portability [1-10]. These methods have shown great potential for human and animal functional studies when integrated with other imaging modalities such as magnetic resonance imaging, which provides structural information and enables improved signal localization and accurate image registration [11-17].

In human functional studies, NIR techniques are most often applied to measure the hemodynamic response in the brain, which peaks approximately four to six seconds after the actual neuronal response via measurement of the change in tissue absorption coefficient $\left(\mu_{a}\right)$. It has also been shown that "fast" optical signals $[18,19]$ can be measured using frequency-domain techniques. This fast signal is derived from the change in signal modulation phase and is believed to be associated with the reduced scattering coefficient $\left(\mu_{s}^{\prime}\right)$. A number of groups have reported fast signals in noninvasive human studies in terms of the changes in either the phase or the intensity of the optical signal [20-23]. Although invasive human and animal studies have demonstrated close coupling of the fast optical signal and neuronal response [19,24-26], detectability of the fast signal in non-invasive human studies is challenging [27]. This is primarily because the signal-to-noise ratio (SNR) of the fast signal is much lower than that of the conventional hemodynamics-induced signal.

To address the issue of signal detectability, we investigated the relative detection limits of the AC and DC components in frequency-domain measurements with respect to $\Delta \mu_{a}$ and the phase change corresponding to $\Delta \mu_{s}^{\prime}$ in an imaging phantom. Similar to the concept of "contrast-detail analysis" for optical imaging described in [28], we characterized the detection limits using three parameters: the amplitude, the size, and the depth of the simulated activation. To the best of our knowledge, this type of data is not available in the literature, which is highly important to understand the fundamental characteristics of frequency-domain NIR methods (including spectroscopy and tomography) in human cerebral functional studies.

\section{Experimental Section}

Several groups have reported imaging phantoms for NIR studies, e.g., [29-34]: in most of those phantoms, different types of absorbers (e.g., ultra-fine carbon powder, ink, or dye) and scattering materials (e.g., titanium dioxide, polystyrene micro-spheres, fat emulsion, or milk) were typically dispersed in rigid, deformable, or liquid media (e.g., silicone, paraffin, polyvinyl alcohol, or water). As shown in the studies by Gibson et al. $[29,35]$, realistic head phantoms are less susceptible to modeling errors arising from the cerebrospinal fluid (CSF) layer than simple semi-infinite or spherical homogenous phantoms.

We constructed a realistic human head phantom from a life-sized mannequin head (Figure 1). The outer layer of the phantom was covered in cosmetically tinted silicone with a thickness of $3 \mathrm{~mm}$, which simulated the scalp. Its optical properties were measured to be $\mu_{a}=0.015$ and $\mu_{s}^{\prime}=1.3 \mathrm{~mm}^{-1}$ at $690 \mathrm{~nm}$, determined iteratively using a two-layered model as described in [36]. A layer of silicone (semi-translucent due to micro-bubbles in the material) was coated on the inner surface of the head 
phantom to simulate the skull and the CSF collectively. This layer of silicone had an intentionally created rough inner surface (with irregular ripples and ridges) with a thickness ranging from 2 to $6 \mathrm{~mm}$.

Figure 1. Schematic of the head phantom and experimental setup.

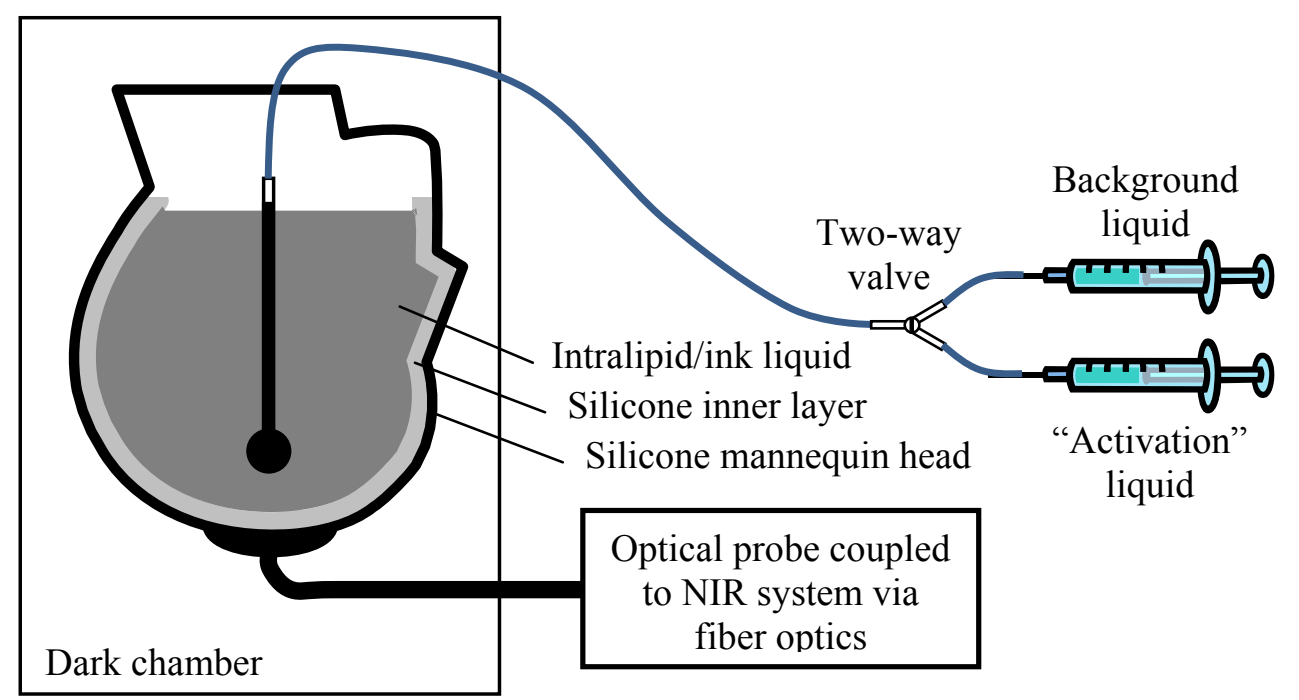

The brain tissue was simulated using a liquid phantom consisting of $1 \%$ Intralipid (VWR, West Chester, PA, USA), 60 ppm India ink, and water. The optical properties of the liquid phantom and the semi-translucent silicone layer were measured using the method detailed in [37]: $\mu_{a}=0.011$ and $\mu_{s}^{\prime}=1.4 \mathrm{~mm}^{-1}$ for the liquid; and $\mu_{a}=0.0017$ and $\mu_{s}^{\prime}=0.11 \mathrm{~mm}^{-1}$ for the silicone layer at $690 \mathrm{~nm}$. The optical probe was positioned on the head phantom at the location corresponding to the primary sensorimotor cortex. During the experiments, the head phantom was kept in a dark chamber.

Figure 2. The optical probe consisted of sixteen source and seven detector fibers that were fixed in a slightly curved rubber base: (a) the triangular topology of the fibers: the larger/blue circles represent the detectors and the smaller/red circles the sources; and (b) a histogram of the source-detector distance distribution.
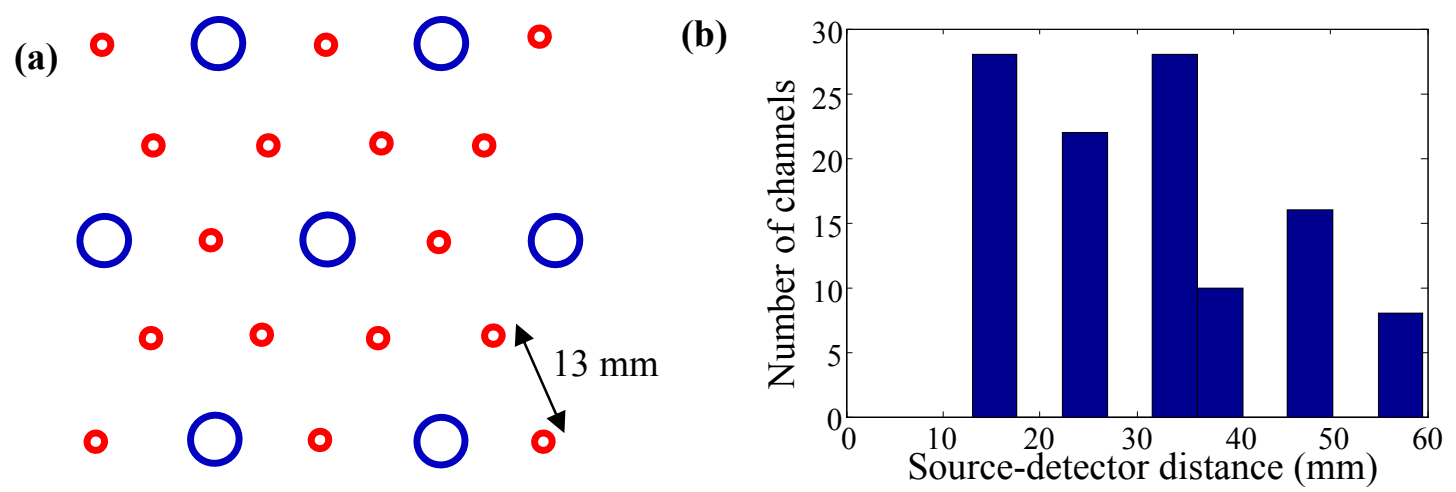

The optical probe was constructed by fixing the source and the detector fibers to a curved black rubber base. The rubber base was attached a hard plastic frame, thereby maintaining its shape and sour-detector separations. The topology of the fibers was designed to represent a typical functional 
diffuse optical tomography (DOT) experiment in which an even coverage of the region of interest (ROI) would be desired. Sixteen source fibers (each coupled to a 690-nm laser diode) and seven detector fibers bundles (each coupled to a photomultiplier tube) were arranged in a triangular pattern, Figure 2(a). The source-detector distances of the optical probe ranged from 13 to $60 \mathrm{~mm}$, Figure 2(b).

We used a frequency-domain NIR spectroscopy/imaging system (Imagent, ISS, Champaign, IL, USA). The light sources were laser diodes (690 nm), which were time-multiplexed, amplitude-modulated (150 MHz), and coupled to multimode fiber optics (core size $400 \mu \mathrm{m}$, Thorlabs, Newton, NJ, USA). The detection fiber optic bundles (diameter $3 \mathrm{~mm}$, numerical aperture 0.55, Sunoptic Technologies, Jacksonville, FL, USA) were coupled to the photomultiplier tubes (PMT), where the optical signal was converted into an electrical signal. The electrical signal was then demodulated using a heterodyne method with a cross-correlation frequency of $10 \mathrm{kHz}$, and subsequently digitized by a 16-bit analog-todigital converter (ADC) (PCI-416M2, DATEL, Mansfield, MA, USA). The effective sampling rate for each measurement channel was $10 \mathrm{~Hz}$.

We simulated functional activation using thin-walled quartz spheres (Wilmad Lab Glass, Buena, NJ, USA). Baseline condition was simulated by filling the spheres with the background liquid phantom, whereas activation condition was simulated by filling with the same type of liquid phantom but with altered optical properties by adjusting the amount of ink and Intralipid. The temporal feature of the functional activation was simulated by switching between the background and altered liquids. Five spheres of different volumes $(0.8,2.1,5.2,11$, and $20 \mathrm{~mL}$, i.e., inner diameters $12,16,21,28$, and $34 \mathrm{~mm}$ ) were placed at different distances, which were measured from the bottom of the sphere to the outer surface of the head phantom, in 2-mm increments. One sphere was placed in the head phantom in any given measurement. Two syringes were connected to a two-way valve and used to fill and drain the sphere: one with background liquid for baseline and another with altered optical properties for activation (Figure 1). Human cerebral functional activation was simulated by switching the liquids every $10 \mathrm{~s}$ for a total of 20 times, i.e., 10 activations and 11 resting intervals interleaved in a total experimental time of $210 \mathrm{~s}$. Similar experimental paradigms are typically used in human studies because it was an appropriate compromise between the amount of acquired data and minimizing subject motion.

For each source-detector measurement channel, the data were first folding-averaged and then processed using the following criteria to determine the detectability: (a) the absolute value of the correlation coefficient of the averaged signal (300 time-series sampling points) and the boxcar activation pattern should be greater than 0.3 (equivalent to a significance value of $p<2 \times 10^{-7}$, according to [38]); and (b) the SNR of the processed signal should be $>0.5$. The SNR was defined as the ratio of the relative change in signal intensity to the standard deviation of the baseline signal. In this study, the detectability was investigated based on the raw data, i.e., the AC, DC, and phase signals. Activation was considered detectable for a given configuration if at least one measurement channel met both of the above criteria.

We used five different liquids that produced 5, 10, 15, 20, and 30\% increase of $\mu_{a}$ with respect to the background. This range of $\Delta \mu_{a}$ values was chosen to cover the normal physiological conditions derived from published experimental data [12,17,39-47] and values adopted by other phantom and simulation experiments pertaining to human functional studies [48-52]. We did not find any experimental data regarding the values of $\Delta \mu^{\prime}{ }_{s}$ for functional brain studies in the literature. In a 
simulation study, the authors estimated the value of $\Delta \mu_{s}^{\prime}$ being less than $0.4 \%$ using a "proportionality factor" assuming a semi-infinite or layered medium [27]. Reported experimental measurements of the phase delay in fast signal measurement range from 0.7 to $10 \mathrm{ps}$, as summarized by Steinbrink et al. [27], and would correspond to much larger values of $\Delta \mu_{s}^{\prime}$. It should be noted that values of the phase delay are very sensitive to the size and the depth of the activation, as well as the sensitivity profile of the optical probe, which should be taken into consideration when comparing experimental results from different subjects and in different cortical areas. In our phantom study we used values of $\Delta \mu_{s}^{\prime}(10,20$, 30,40 , and $50 \%$ increase from baseline) that produced detectable changes in the phase of the optical signal for the same size and depth of activation, sampling rate, and the number of measurements used to measure the detectability of $\Delta \mu_{a}$.

\section{Results and Discussion}

The maximum detectable depth is plotted against the relative value of $\Delta \mu_{a}$ (percentage change) for different activation volumes in terms of the $\mathrm{AC}$ and the DC signals, Figure 3(a,b), respectively. It was obtained by incrementally increasing the depth of activation, measured from the bottom of the inner surface of the sphere to the outer surface of the phantom, until the change in $\mu_{a}$ or $\mu_{s}^{\prime}$ was undetectable according to the criteria described previously. As expected, the detectable depth of activation increases as the size and the amplitude of change increase. It is noteworthy that because the sensitivity function is narrower for superficial measurement channels than deeper channels, the depth- $\Delta \mu_{a}$ curves appear non-linear. This effect is more apparent for larger spheres than smaller ones.

Figure 3. Detectability of the changes in absorption coefficient $\mu_{a}$ in terms of (a) the AC signal and (b) the DC signal, with respect to the background (baseline) value.
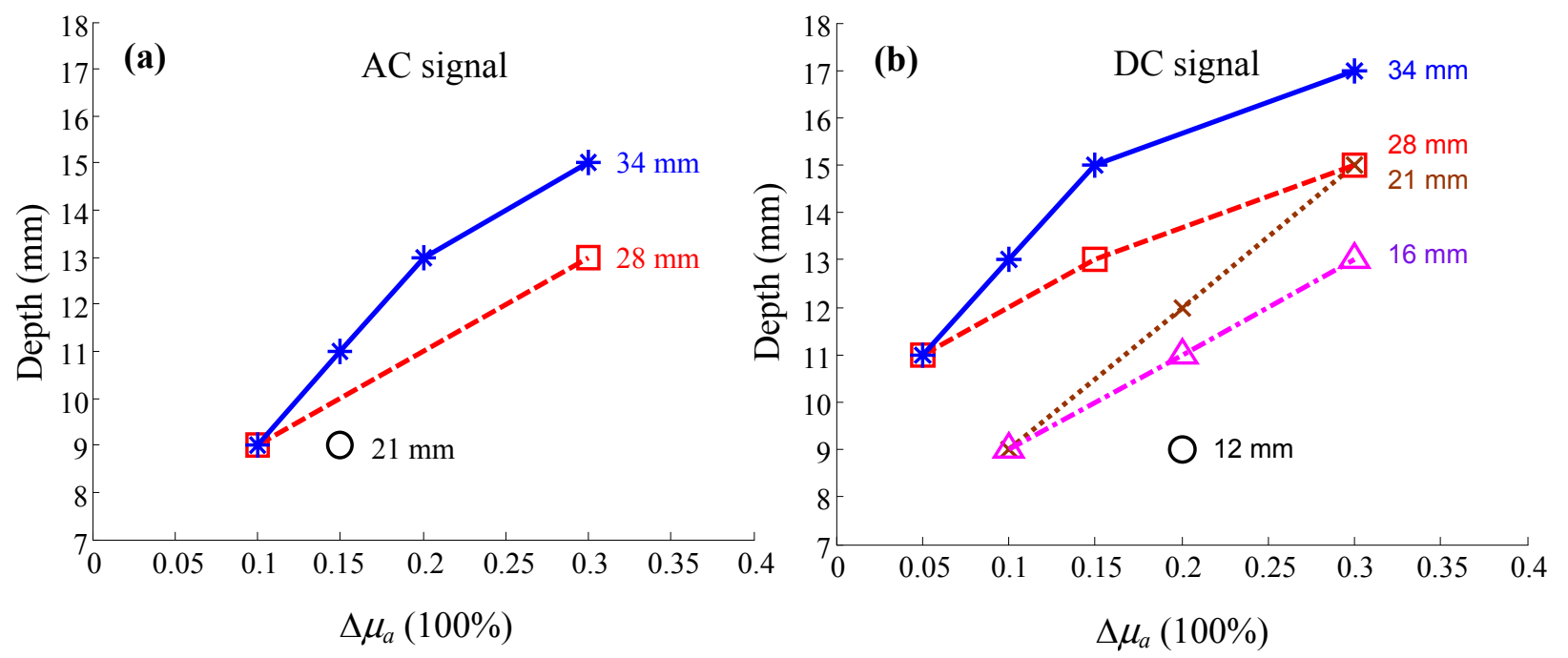

Following the theoretical analysis in $[53,54]$, the first-order Born solution of the diffusion equation is given by:

$$
\delta U=-\int_{\Omega} \delta \mu_{a} G_{0} U_{0} d \Omega
$$


where $U_{0}$ is the fluence of the light source (i.e., the field of light radiation) in the baseline condition, $G_{0}$ is the Green function, $U$ is the detected photon fluence rate, and $\Omega$ is the integral volume. It shows that the change in the optical signal is linearly related to $\Delta \mu_{a}$ if scattering can be ignored: this corresponds to measurement of the hemodynamic optical signal, as described previously.

The SNR of the optical signal is linearly related to the amplitude, but its relationship to the volume and the depth of $\Delta \mu_{a}$ is non-linear since the integral kernel $G_{0} U_{0}$ is spatially inhomogeneous. This is evident in the data shown in Figure 3, in which the dependence of the maximum detectable depth (y-axis) on the minimum detectable $\Delta \mu_{a}$ (x-axis) is non-linear for a given activation volume. However, such non-linearity stems from the non-uniform distribution of the sensitivity function. For smaller spheres at smaller depth, the non-uniformity is much less than that of larger spheres at larger depth. As a result, the depth-detectability relations for smaller spheres (also smaller detectable depth) appear linear under our experimental conditions.

Comparing Figure 3(a,b), the result indicates that activations are more sensitive using the DC signal than using the AC signal: smaller volumes of activation being detectable using the DC signal; and the maximum detectable depth using the DC signal being approximately $2-3 \mathrm{~mm}$ larger than using the AC signal for a given volume.

Another observation from Figure 3 is that, although the detectable depth using the DC signal is only slightly larger than that using the AC signal, the DC signal is capable of detecting much smaller activations in our experimental setup: activation volumes of 0.8 and $2.1 \mathrm{~mL}$ were not detectable using the AC signal even at the smallest depth (10 and $12 \mathrm{~mm}$ respectively from the bottom of the inner surface of the activation to the probe/phantom interface).

Although absolute values of detectability of the AC and DC signals depend upon the particular measurement system, the frequency dependence, for example, of relative detectability can be estimated by considering the noise contributions to each measurement. A schematic of our NIR system is shown in Figure 4. In phantom experiments, the only source of noise is "system noise" which has several contributors: quantum noise from the photodetector when the intensity of light is low, dark noise due to the dark current in the photodetector (PMT), thermal noise from electronics (particularly the signal amplifiers), quantization noise from the $\mathrm{ADC}$, temperature drift (causing the changes in the semiconductor characteristics and the level of thermal noise) as well as the fluctuation of the voltage/current supplies of the PMT and the laser diodes. The noise level at the input to the ADC is dictated by the characteristics of each electronic component. If one assumes that the noise figure and the gain of the amplifiers are frequency-independent across the measurement bandwidth $(10 \mathrm{kHz}$ in this study), then the system noise level seen by the ADC is the same for both the AC and the DC measurements since the two measures are not separated until after quantization at the ADC. In our system, the noise in the AC and the DC signals is dominated by the analogue data acquisition subsystem, because the amplifier gain is high, the noise figure is relatively low, and the high digital resolution of the 16-bit ADC (a lower limit of the quantization error of $<1 \times 10^{-5}$ ).

The relative SNR of the AC and the DC signals are therefore determined both by their relative intensities and relative sensitivities. In terms of relative intensities, the modulation depth (defined as the ratio of $\mathrm{AC}$ to $\mathrm{DC}$ signals) of the NIR system used in our experiment was $50 \%$, meaning that the $\mathrm{DC}$ signal is twice as high as the AC at the source. The relative sensitivity depends on the modulation frequency of the instrumentation. The frequency-dependence of the sensitivity function for a semi- 
infinite medium (using the analytical expression in [55]) is shown in Figure 5. For a source-detector separation of $20 \mathrm{~mm}$, at a modulation frequency of $150 \mathrm{MHz}$, and at a depth of $20 \mathrm{~mm}$ from the surface mid-way between the source and the detector, the absolute value of the sensitivity function is $88 \%$ of its value at DC. The above analysis indicates that at $150 \mathrm{MHz}$ modulation, the DC signal should be $(50 \% \times 88 \%)^{-1}=2.3$ times more sensitive than the AC signal in terms of SNR. Note that at higher modulation frequencies, this advantage will increase. In practice, one also has to consider that at higher frequencies electronic components typically have lower gain and higher noise figure.

Figure 4. Schematic of the frequency-domain NIR system, where $F_{\text {in }}$ is the modulation frequency and $f_{c c}$ is the cross-correlation frequency.

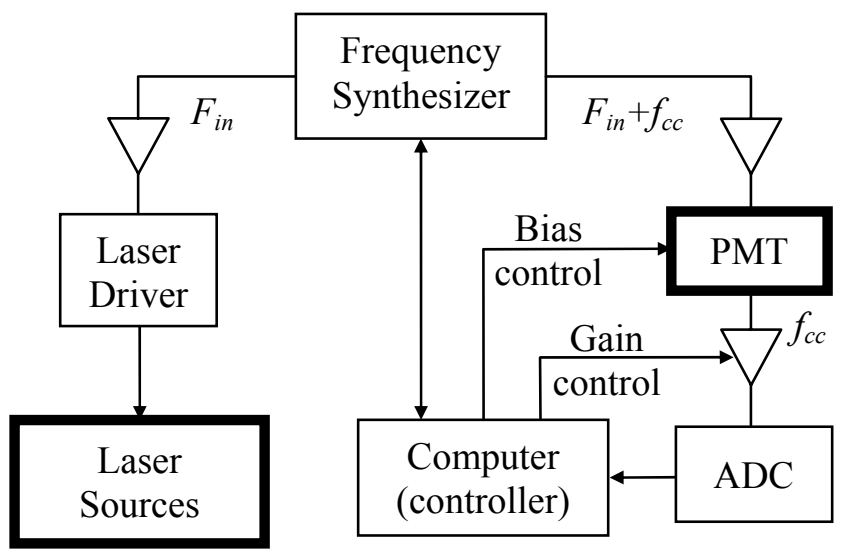

Figure 5. Frequency-dependence of relative sensitivity for a semi-infinite medium $\left(\mu_{a}=0.01\right.$ and $\mu_{s}^{\prime}=1 \mathrm{~mm}^{-1}$ ) with a source-detector distance of $20 \mathrm{~mm}$ at a depth of $20 \mathrm{~mm}$.

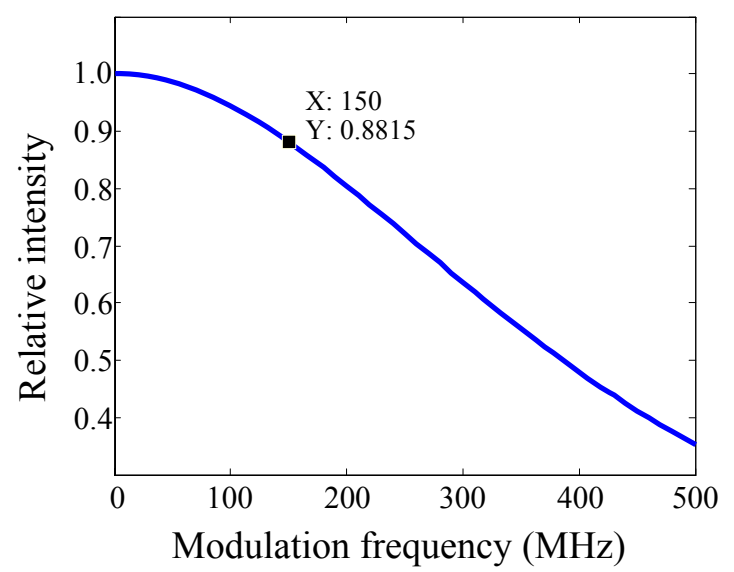

The detectability of $\Delta \mu_{s}^{\prime}$ in terms of changes in the phase signal is shown in Figure 6(a). Both $\Delta \mu_{a}$ and $\Delta \mu_{s}^{\prime}$ result in changes in the AC and the DC signals, but the phase signal is solely dependent on $\Delta \mu_{s}^{\prime}$ : a significant advantage of the frequency-domain measurement method [56]. The detectability of $\Delta \mu_{s}^{\prime}$ shows similar characteristics to that of $\Delta \mu_{a}$. However, comparison between Figures 3 and 6(a) shows that, to achieve a similar maximal detectable depth, the amplitude of $\Delta \mu_{s}^{\prime}$ has to be larger than that of $\Delta \mu_{a}$ by a factor of $\sim 2$.

In order to understand the shape of the graph in Figure 6, we note that the first-order Rytov solution to the diffusion equation: 


$$
\delta \Phi=\frac{1}{U_{0}} \int_{\Omega} \delta D\left(\nabla G_{0} \cdot \nabla U_{0}\right) d \Omega
$$

in which $\Phi$ is defined as $\ln (U)$. It shows that $\delta \Phi$ is linearly related to the change in the light diffusion coefficient $D$, if the changes in the absorption coefficient are ignored (a reasonable assumption since immediately after neuronal activation the hemodynamic response has not yet developed). As the fluence is complex and can be written in the form of $U=A \cdot e^{j \theta}$, it follows that:

$$
\delta \Phi=\ln (U)-\ln \left(U_{0}\right)=\ln \left(A \cdot e^{j \theta}\right)-\ln \left(A_{0} \cdot e^{j \theta_{0}}\right)=\ln \left(\frac{A}{A_{0}}\right)+j\left(\theta-\theta_{0}\right)
$$

Since the diffusion coefficient can be defined as $D=\left(3 \mu_{s}^{\prime}\right)^{-1}$, the dependence of the phase delay of the optical signal should be a linear function of the reciprocal of $\mu_{s}^{\prime}$ in the form of $y=a\left(1-x^{-1}\right)$, where $y$ is the phase delay, $x$ is the normalized value of $\mu_{s}^{\prime}$ (by its baseline value, $1<x<2$ ), and $a$ is a constant. Figure 6(b) shows good agreement between the experimentally measured phase delay (34 $\mathrm{mm}$ sphere at depth $11 \mathrm{~mm}$ ) and the theoretical prediction (the fitted value of 0.22 in this instance).

Figure 6. (a) Detectability of the change in the reduced scattering coefficient from the phase signal and (b) relationship between the changes in the phase signal and the values of the reduced scattering coefficient revealed in experimental data and theoretical analysis.
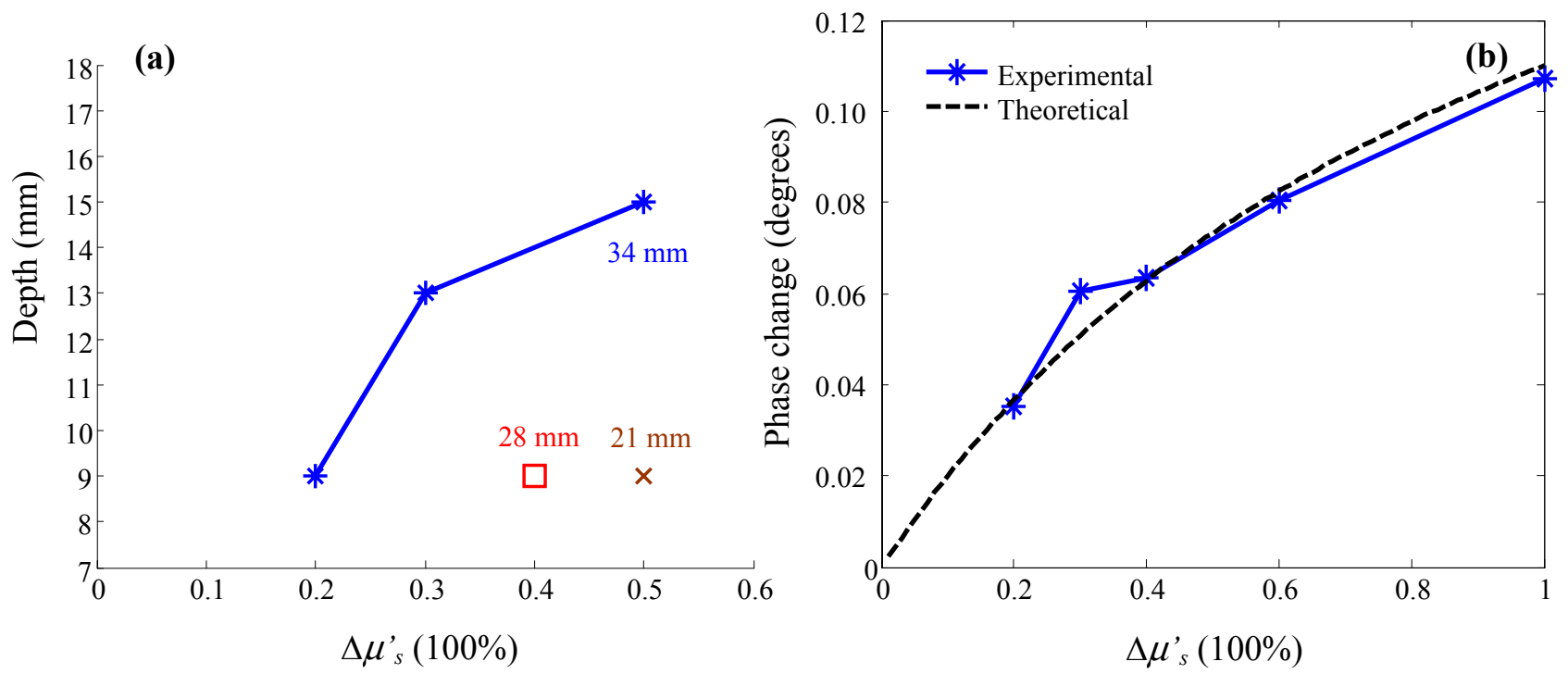

Detectability of $\Delta \mu_{s}^{\prime}$ is fundamentally limited by the SNR of the phase signal. The dominant noise source for phase measurement is the time-jitter of the ADC, which is caused by instabilities in the core frequency source (master oscillator). In our system, the ADC used an external frequency synthesizer (D620, PTS, Littleton, MA, USA) as the frequency source $(10 \mathrm{MHz} \pm 2 \mathrm{~Hz})$. For a cross-correlation frequency of $10 \mathrm{kHz}$, this tolerance is equivalent to a peak-to-peak phase jitter of $\pm 0.72^{\circ}$, which corresponds to a standard deviation of $0.42^{\circ}$ if the jitter is truly random. Figure 7 shows a histogram of the standard deviation of the phase signal from a representative measurement. About $65 \%$ of the measurement channels had standard deviations equal or less than the error limit estimated above. Even the largest value is only twice the estimated error limit. 
Figure 7. A histogram of the standard deviation of the phase signal from a representative measurement. The channels that had AC signals less than 5\% of the maximum value were excluded to eliminate unreliable phase measurements due to small signal intensity.

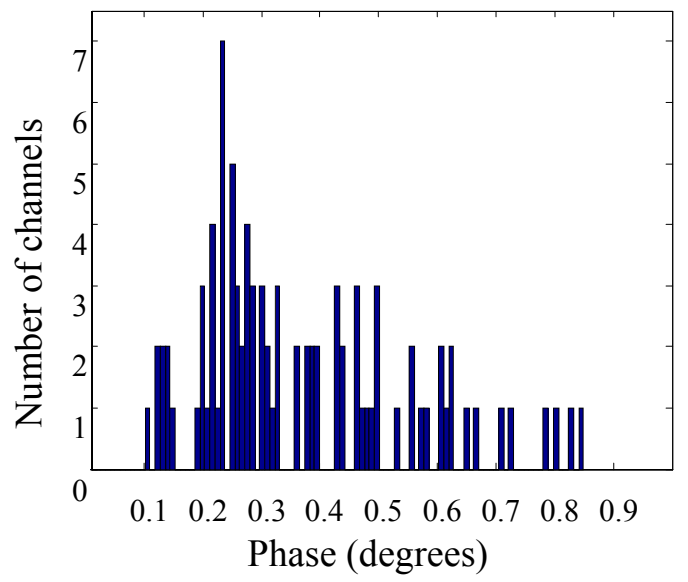

To ensure the validity of our general conclusions, we intentionally chose a set of relatively conservative criteria to define the detectability. As a result, some measurements were determined undetectable by these particular criteria, but were in fact detectable by visual inspection. In addition, the number of valid measurement channels had impact on the detectability. Our experiment had 112 measurement channels (16 sources and 7 detectors). The number of valid channels for superficial activations (i.e., short distance) is significantly larger than deep activations (longer distance). As a result, these limitations influenced the observed detectability by means of introducing false negative in the results (omitting detectable activation). However, the data sets that passed the relative conservative detectability criteria are robust and well support the general conclusions based upon them.

\section{Conclusions}

We have presented results using a life-size head phantom with realistic optical properties for studying the measurement sensitivity of absorption and reduced scattering coefficients. The detectability of simulated functional activation was quantified in terms of the maximum detectable depth for absorption and scattering changes of different amplitudes and volumes ranging 0.8-20 microliters using a frequency-domain NIR system with a modulation frequency of $150 \mathrm{MHz}$. The non-linear relation between the maximum detectable depth and the magnitude of changes in the absorption coefficient conform well with the Born approximation to the diffusion equation. The amplitude of the experimentally measured phase delay was found to be linearly related to the reciprocal of $\mu_{s}^{\prime}$ as predicted by the first-order Rytov solution to the diffusion equation. For the same volume and depth of activation, the minimum detectable amplitude of $\Delta \mu_{s}^{\prime}$ is approximately twice as large as that of $\Delta \mu_{a}$.

\section{Acknowledgments}

This project was supported by the National Center for Research Resources and the National Institute of Biomedical Imaging and Bioengineering of the National Institutes of Health through grant numbers R21RR025824 and R21EB015907. 


\section{References}

1. Chance, B.; Luo, Q.; Nioka, S.; Alsop, D.C.; Detre, J.A. Optical investigations of physiology: A study of intrinsic and extrinsic biomedical contrast. Philos. Trans. Roy. Soc. Lond B Biol. Sci. 1997, 352, 707-716.

2. Obrig, H.; Villringer, A. Beyond the visible-Imaging the human brain with light. J. Cereb Blood Flow Metab 2003, 23, 1-18.

3. Toronov, V.; Walker, S.; Gupta, R.; Choi, J.H.; Gratton, E.; Hueber, D.; Webb, A. The roles of changes in deoxyhemoglobin concentration and regional cerebral blood volume in the fMRI BOLD signal. Neuroimage 2003, 19, 1521-1531.

4. Gratton, E.; Toronov, V.; Wolf, U.; Wolf, M.; Webb, A. Measurement of brain activity by near-infrared light. J. Biomed. Opt. 2005, 10, 11008.

5. Franceschini, M.A.; Joseph, D.K.; Huppert, T.J.; Diamond, S.G.; Boas, D.A. Diffuse optical imaging of the whole head. J. Biomed. Opt. 2006, 11, 054007.

6. Sassaroli, A.; deB. Frederick, B.; Tong, Y.; Renshaw, P.F.; Fantini, S. Spatially weighted BOLD signal for comparison of functional magnetic resonance imaging and near-infrared imaging of the brain. Neuroimage 2006, 33, 505-514.

7. Brown, J.Q.; Wilke, L.G.; Geradts, J.; Kennedy, S.A.; Palmer, G.M.; Ramanujam, N. Quantitative optical spectroscopy: A robust tool for direct measurement of breast cancer vascular oxygenation and total hemoglobin content in vivo. Cancer Res. 2009, 69, 2919-2926.

8. Fu, H.L.; Yu, B.; Lo, J.Y.; Palmer, G.M.; Kuech, T.F.; Ramanujam, N. A low-cost, portable, and quantitative spectral imaging system for application to biological tissues. Opt. Express 2010, 18, 12630-12645.

9. Arridge, S.R. Methods in diffuse optical imaging. Philos. Trans. A Math. Phys. Eng. Sci. 2011, $369,4558-4576$.

10. Gurley, K.; Shang, Y.; Yu, G. Noninvasive optical quantification of absolute blood flow, blood oxygenation, and oxygen consumption rate in exercising skeletal muscle. J. Biomed. Opt. 2012, 17, 075010.

11. Kleinschmidt, A.; Obrig, H.; Requardt, M.; Merboldt, K.D.; Dirnagl, U.; Villringer, A.; Frahm, J. Simultaneous recording of cerebral blood oxygenation changes during human brain activation by magnetic resonance imaging and near-infrared spectroscopy. J. Cereb. Blood Flow Metab. 1996, $16,817-826$.

12. Mehagnoul-Schipper, D.J.; van der Kallen, B.F.; Colier, W.N.; van der Sluijs, M.C.; van Erning, L.J.; Thijssen, H.O.; Oeseburg, B.; Hoefnagels, W.H.; Jansen, R.W. Simultaneous measurements of cerebral oxygenation changes during brain activation by near-infrared spectroscopy and functional magnetic resonance imaging in healthy young and elderly subjects. Human Brain Map. 2002, 16, 14-23.

13. Strangman, G.; Culver, J.P.; Thompson, J.H.; Boas, D.A. A quantitative comparison of simultaneous BOLD fMRI and NIRS recordings during functional brain activation. Neuroimage 2002, 17, 719-731. 
14. Okamoto, M.; Dan, H.; Shimizu, K.; Takeo, K.; Amita, T.; Oda, I.; Konishi, I.; Sakamoto, K.; Isobe, S.; Suzuki, T.; Kohyama, K.; Dan, I. Multimodal assessment of cortical activation during apple peeling by NIRS and fMRI. Neuroimage 2004, 21, 1275-1288.

15. Gulsen, G.; Birgul, O.; Unlu, M.B.; Shafiiha, R.; Nalcioglu, O. Combined diffuse optical tomography (DOT) and MRI system for cancer imaging in small animals. Technol. Cancer Res. Treat. 2006, 5, 351-363.

16. Schroeter, M.L.; Kupka, T.; Mildner, T.; Uludag, K.; von Cramon, D.Y. Investigating the post-stimulus undershoot of the BOLD signal-A simultaneous fMRI and fNIRS study. Neuroimage 2006, 30, 349-358.

17. Toronov, V.Y.; Zhang, X.; Webb, A.G. A spatial and temporal comparison of hemodynamic signals measured using optical and functional magnetic resonance imaging during activation in the human primary visual cortex. Neuroimage 2007, 34, 1136-1148.

18. Gratton, G.; Fabiani, M. The event-related optical signal (EROS) in visual cortex: Replicability, consistency, localization, and resolution. Psychophysiology 2003, 40, 561-571.

19. Medvedev, A.V.; Kainerstorfer, J.M.; Borisov, S.V.; Gandjbakhche, A.H.; Vanmeter, J. "Seeing" electroencephalogram through the skull: Imaging prefrontal cortex with fast optical signal. J. Biomed. Opt. 2010, 15, 061702.

20. Steinbrink, J.; Kohl, M.; Obrig, H.; Curio, G.; Syre, F.; Thomas, F.; Wabnitz, H.; Rinneberg, H.; Villringer, A. Somatosensory evoked fast optical intensity changes detected non-invasively in the adult human head. Neurosci. Lett. 2000, 291, 105-108.

21. Wolf, M.; Wolf, U.; Choi, J.H.; Gupta, R.; Safonova, L.P.; Paunescu, L.A.; Michalos, A.; Gratton, E. Functional frequency-domain near-infrared spectroscopy detects fast neuronal signal in the motor cortex. Neuroimage 2002, 17, 1868-1875.

22. Tong, Y.; Martin, J.M.; Sassaroli, A.; Clervil, P.R.; Bergethon, P.R.; Fantini, S. Fast optical signals in the peripheral nervous system. J. Biomed. Opt. 2006, 11, 044014.

23. Tse, C.Y.; Tien, K.R.; Penney, T.B. Event-related optical imaging reveals the temporal dynamics of right temporal and frontal cortex activation in pre-attentive change detection. Neuroimage 2006, 29, 314-320.

24. Rector, D.M.; Poe, G.R.; Kristensen, M.P.; Harper, R.M. Light scattering changes follow evoked potentials from hippocampal Schaeffer collateral stimulation. J. Neurophysiol. 1997, 78, 1707-1713.

25. Rector, D.M.; Carter, K.M.; Volegov, P.L.; George, J.S. Spatio-temporal mapping of rat whisker barrels with fast scattered light signals. Neuroimage 2005, 26, 619-627.

26. Suh, M.; Bahar, S.; Mehta, A.D.; Schwartz, T.H. Blood volume and hemoglobin oxygenation response following electrical stimulation of human cortex. Neuroimage 2006, 31, 66-75.

27. Steinbrink, J.; Kempf, F.C.; Villringer, A.; Obrig, H. The fast optical signal-Robust or elusive when non-invasively measured in the human adult? Neuroimage 2005, 26, 996-1008.

28. Pogue, B.W.; Davis, S.C.; Song, X.; Brooksby, B.A.; Dehghani, H.; Paulsen, K.D. Image analysis methods for diffuse optical tomography. J. Biomed. Opt. 2006, 11, 33001.

29. Gibson, A.; Yusof, R.M.; Dehghani, H.; Riley, J.; Everdell, N.; Richards, R.; Hebden, J.C.; Schweiger, M.; Arridge, S.R.; Delpy, D.T. Optical tomography of a realistic neonatal head phantom. Appl. Opt. 2003, 42, 3109-3116. 
30. Liu, N.; Sassaroli, A.; Fantini, S. Two-dimensional phased arrays of sources and detectors for depth discrimination in diffuse optical imaging. J. Biomed. Opt. 2005, 10, 051801.

31. Passos, D.; Hebden, J.C.; Pinto, P.N.; Guerra, R. Tissue phantom for optical diagnostics based on a suspension of microspheres with a fractal size distribution. J. Biomed. Opt. 2005, 10, 064036.

32. Pogue, B.W.; Patterson, M.S. Review of tissue simulating phantoms for optical spectroscopy, imaging and dosimetry. J. Biomed. Opt. 2006, 11, 041102.

33. Rao, K.P.; Radhakrishnan, S.; Reddy, M.R. Brain tissue phantoms for optical near infrared imaging. Exper. Brain Res. Exper. Hirnforsch. Exper. Cereb. 2006, 170, 433-437.

34. Hebden, J.C.; Price, B.D.; Gibson, A.P.; Royle, G. A soft deformable tissue-equivalent phantom for diffuse optical tomography. Phys. Med. Biol. 2006, 51, 5581-5590.

35. Gibson, A.P.; Hebden, J.C.; Riley, J.; Everdell, N.; Schweiger, M.; Arridge, S.R.; Delpy, D.T. Linear and nonlinear reconstruction for optical tomography of phantoms with nonscattering regions. Appl. Opt. 2005, 44, 3925-3936.

36. Kienle, A.; Patterson, M.S.; Dognitz, N.; Bays, R.; Wagninures, G.; van den Bergh, H. Noninvasive determination of the optical properties of two-layered turbid media. Appl. Opt. 1998, 37, 779-791.

37. Fantini, S.; Franceschini, M.A.; Fishkin, J.B.; Barbieri, B.; Gratton, E. Quantitative determination of the absorption spectra of chromophores in strongly scattering media: A light-emitting-diode based technique. Appl. Opt. 1994, 33, 5204-5213.

38. Bandettini, P.A.; Jesmanowicz, A.; Wong, E.C.; Hyde, J.S. Processing strategies for time-course data sets in functional MRI of the human brain. Magn. Resona. Med. 1993, 30, 161-173.

39. Franceschini, M.A.; Toronov, V.; Filiaci, M.; Gratton, E.; Fantini, S. On-line optical imaging of the human brain with 160-ms temporal resolution. Opt. Express 2000, 6, 49-57.

40. Toronov, V.; Franceschini, M.A.; Filiaci, M.; Fantini, S.; Wolf, M.; Michalos, A.; Gratton, E. Near-infrared study of fluctuations in cerebral hemodynamics during rest and motor stimulation: temporal analysis and spatial mapping. Med. Phys. 2000, 27, 801-815.

41. Takahashi, K.; Ogata, S.; Atsumi, Y.; Yamamoto, R.; Shiotsuka, S.; Maki, A.; Yamashita, Y.; Yamamoto, T.; Koizumi, H.; Hirasawa, H.; Igawa, M. Activation of the visual cortex imaged by 24-channel near-infrared spectroscopy. J. Biomed. Opt. 2000, 5, 93-96.

42. Lee, S.P.; Duong, T.Q.; Yang, G.; Iadecola, C.; Kim, S.G. Relative changes of cerebral arterial and venous blood volumes during increased cerebral blood flow: Implications for BOLD fMRI. Magn. Resona. Med. 2001, 45, 791-800.

43. Wolf, M.; Wolf, U.; Choi, J.H.; Toronov, V.; Paunescu, L.A.; Michalos, A.; Gratton, E. Fast cerebral functional signal in the 100-ms range detected in the visual cortex by frequency-domain near-infrared spectrophotometry. Psychophysiology 2003, 40, 521-528.

44. Buxton, R.B.; Uludag, K.; Dubowitz, D.J.; Liu, T.T. Modeling the hemodynamic response to brain activation. Neuroimage 2004, 23(Suppl 1), S220-S233.

45. Hoge, R.D.; Franceschini, M.A.; Covolan, R.J.; Huppert, T.; Mandeville, J.B.; Boas, D.A. Simultaneous recording of task-induced changes in blood oxygenation, volume, and flow using diffuse optical imaging and arterial spin-labeling MRI. Neuroimage 2005, 25, 701-707. 
46. Selb, J.; Stott, J.J.; Franceschini, M.A.; Sorensen, A.G.; Boas, D.A. Improved sensitivity to cerebral hemodynamics during brain activation with a time-gated optical system: analytical model and experimental validation. J. Biomed. Opt. 2005, 10, 11013.

47. Gatto, R.; Hoffman, W.; Mueller, M.; Flores, A.; Valyi-Nagy, T.; Charbel, F.T. Frequency domain near-infrared spectroscopy technique in the assessment of brain oxygenation: A validation study in live subjects and cadavers. J. Neurosci. Methods 2006, 157, 274-277.

48. Kawaguchi, H.; Hayashi, T.; Kato, T.; Okada, E. Theoretical evaluation of accuracy in position and size of brain activity obtained by near-infrared topography. Phys. Med. Biol. 2004, 49, 2753-2765.

49. Srinivasan, S.; Pogue, B.W.; Dehghani, H.; Jiang, S.; Song, X.; Paulsen, K.D. Improved quantification of small objects in near-infrared diffuse optical tomography. J. Biomed. Opt. 2004, 9, 1161-1171.

50. Intes, X.; Maloux, C.; Guven, M.; Yazici, B.; Chance, B. Diffuse optical tomography with physiological and spatial a priori constraints. Phys. Med. Biol. 2004, 49, N155-N163.

51. Zhang, Y.; Brooks, D.H.; Boas, D.A. A haemodynamic response function model in spatio-temporal diffuse optical tomography. Phys. Med. Biol. 2005, 50, 4625-4644.

52. Corlu, A.; Choe, R.; Durduran, T.; Lee, K.; Schweiger, M.; Arridge, S.R.; Hillman, E.M.; Yodh, A.G. Diffuse optical tomography with spectral constraints and wavelength optimization. Appl. Opt. 2005, 44, 2082-2093.

53. Arridge, S.R.; Cope, M.; Delpy, D.T. The theoretical basis for the determination of optical pathlengths in tissue: Temporal and frequency analysis. Phys. Med. Biol. 1992, 37, 1531-1560.

54. Zhang, X.; Toronov, V.Y.; Webb, A.G. An integrated measurement system for simultaneous functional magnetic resonance imaging and diffuse optical tomography in human brain mapping. Rev. Sci. Instrum. 2006, 77, 114301-1143018.

55. Fantini, S.; Franceschini, M.A.; Gratton, E. Semi-infinite-geometry boundary problem for light migration in highly scattering media: A frequency-domain study in the diffusion approximation. J. Opt. Soc. Am. B 1994, 11, 2128-2138.

56. Arridge, S.R.; Lionheart, W.R.B. Nonuniqueness in diffusion-based optical tomography. Opt. Lett. 1998, 23, 882-884.

(C) 2013 by the authors; licensee MDPI, Basel, Switzerland. This article is an open access article distributed under the terms and conditions of the Creative Commons Attribution license (http://creativecommons.org/licenses/by/3.0/). 